\title{
Rezensionen
}

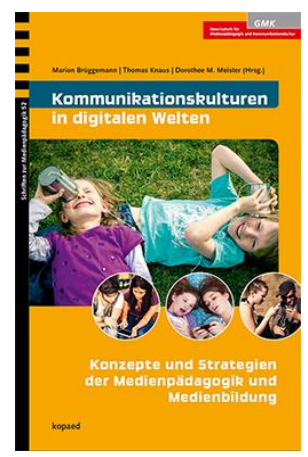

Marion Brüggemann, Thomas Knaus und Dorothee Meister (Hrsg.)

Kommunikationskulturen in digitalen Welten. Konzepte und Strategien der Medienpädagogik und Medienbildung.

Schriftenreihe Schriften zur Medienpädagogik, Band 52

München: kopaed, 2017. 256 Seiten

ISBN: 978-3-86736-552-9 (Print), 9783867369213 (E-Book)

$16,00 €$ (Print), 11,99 $€$ (E-Book) / CHF 23,90 (Print), CHF 15,45 (E-Book)

\section{Neue medienpädagogische Konzepte für das Leben in einer digital geprägten Welt}

Im Vorwort beschreiben die Autorinnen und Autoren digitale Kommunikation als Grundlage und Herausforderung für lebenslang andauernde Bildungsprozesse. Es wird postuliert, dass die Potentiale und Risiken der Entwicklung von Kommunikationskulturen in digitalen Welten gezielte medienpädagogische Konzepte und Strategien erfordern. Die Folgen dieser Entwicklung für Erziehung, Bildung, politische und kulturelle Teilhabe seien nicht abschätzbar. Während die Medienpädagogik sich daher neu orientiert, ist es gleichzeitig ihre Aufgabe, Orientierung zu bieten. Es bedarf neuer angemessener Konzepte, um das Aufwachsen, Leben und Arbeiten in unserer digitalisierten Welt zu gestalten. Die einzelnen Beiträge beinhalten verschiedene Konzepte, welche im Folgenden beschrieben werden.

Im ersten Beitrag von Friedrich Krotz («Wandel von sozialen Beziehungen, Kommunikationskultur und Medienpädagogik») wird zunächst der Medienbegriff thematisiert. Dabei wird eine Definition vorgeschlagen, die universal «für jedes einzelne Medium» gelten soll (S. 22). Nach der Begriffsklärung folgt eine Skizzierung des Medienwandels, bei der nicht einzelne Medien, sondern der simultanen Wandel des gesamten Mediensystems in den Blick genommen wird, wobei Krotz Computer als «zentrales Instrument» der digitalen Infrastruktur betrachtet (S. 25). Der Autor widmet sich dann dem Begriff der Mediatisierung, bei dem es um die zentrale Frage geht, wie der Medienwandel «einen Wandel von Alltag, Kultur und Gesellschaft» bewirkt (S. 27). Dabei werden Veränderungen des Kommunikationsverhaltens, des Machtgefüges gesellschaftlicher Institutionen, inhaltliche Veränderungen und veränderte menschliche Bedürfnisse angesprochen. Es wird herausgestellt, dass Medienwandel und Mediatisierungsprozesse «weder linear [...], noch kausal als Wirkung, und auch nicht auf das Handeln Einzelner zurückgeführt werden» 


\section{Rezensionen}

können, sondern in komplexer Weise miteinander zusammenhängen (S. 28). Der Abschnitt gipfelt in fünf charakteristischen Kernüberlegungen zum Mediatisierungsansatz. Dazu gehört z.B., dass im Rahmen des Mediatisierungsansatzes nicht die «Perspektive der Medien», sondern die "der Menschen» in verschiedenen sozialen Welten untersucht wird (S. 28f.). Schliesslich geht Krotz auf den Wandel jugendlicher Lebensformen ein, insbesondere auf die Veränderung von Habitus und Sozialcharakter menschlicher Akteure im Zusammenhang mit ihrem Medienhandeln. Herausgehoben wird die Mediatisierung von Situationen, in denen zunehmend Daten erhoben würden, die mit dem Körper verbunden sind (z.B. über Krankheit). Es wird auf erhobene Daten (S. 34), das "Universum von Daten» und «Datensammler» eingegangen (S. 35), was der Abschnitt «Wandel durch Kontrolle» vertieft. Es folgt die Thematisierung eines veränderten Informationsverhaltens sowie des Wandels des Subjekts, veränderter Kultur- und Handlungstechniken und Wahrnehmungsweisen. Insgesamt liefert der Beitrag einen guten Einblick in das Thema Mediatisierung. Eine klare, diskutierbare, aber auch anregende Positionierung des Autors wird deutlich. Bei der Darstellung des Wandels jugendlicher Lebensformen spielt das Thema Daten streckenweise eine zentrale Rolle, was nur bedingt der «Perspektive der Menschen» bzw. Jugendlichen entsprechen wird. Dennoch sind gerade die Ausführungen zur Mediatisierung der Situation lesenswert. Der Wandel des Informationsverhaltens und des Subjekts bleibt unkonkret, was der Kürze des Kapitels geschuldet sein kann.

Daniel Süss beschreibt Medien als selbstverständliche «Lebens-, Genussund Suchtmittel» in jugendlichen Lebenswelten. Den Umgang mit Medien gleichzusetzen mit dem Konsum von Nahrungsmitteln, spiegelt diese Selbstverständlichkeit wider und zeigt sich auch in neuen Begriffen wie «HandyFasten» und «Digital Detox». Beim Fasten und Entgiften handelt es sich um «bewusst gesetzte Zäsuren», also «um Formen des Innehaltens». Süss beschreibt wie klassische Ansätze der Psychologie und der Psychopathologie mit dem bewahrpädagogischen Ansatz der Medienpädagogik und einer eher kulturpessimistischen Sichtweise vergleichbar sind, während die Positive Psychologie den Faktor der Resilienz in den Blick genommen hat. Aus dieser Perspektive lassen sich Handlungsempfehlungen für Begleitung und Beratung, zur Förderung von Medienkompetenz als Resilienzfaktor und zur Förderung positiver Medienangebote und Umgangsformen ableiten. Wichtige Schutzfaktoren gegenüber den Risiken in digitalen Welten sind demnach 


\section{Rezensionen}

Selbst-, Sozial- und Medienkompetenz. Medienkompetenz kann dazu beitragen, eine Balance zwischen kommunikativer Beanspruchung und Eigenzeit zu schaffen, «in der Individuen sich selbst wahrnehmen und sich so ausrichten, das Wohlbefinden und Lebensqualität wachsen können» (S. 51).

Büsch und Schreiber postulieren in ihrem Beitrag «Let's talk about Werte. Ethische Herausforderungen für die Medienpädagogik in der digitalisierten Netzwerkgesellschaft» eine Rückbesinnung auf geltende Werte sowie einen «neuen Medienethos» als Aufgabe der Medienpädagogik, weil Medien als Sozialisationsinstanz fungieren und dementsprechend Werte vermitteln. Die Auseinandersetzung mit den Gefahren und Herausforderungen der Digitalisierung wie z.B. Trolling und Hate Speech können ohne einen Rückbezug auf Werte nicht geführt werden. Es bedarf daher einer Medienethik und einer medienpädagogischen Vermittlung von Wertkompetenz durch Medienkompetenz.

Der Titel des Beitrags von Gerhard Tulodziecki lautet «Aktuelle Debatten beim GMK-Forum 2015 im «Rückspiegel〉. Welchen Lösungsbeitrag können medienpädagogische Grundlagen leisten?» Tulodziecki greift darin die medienpädagogischen Diskussionsstränge des GMK-Forums 2015 in Köln auf. Er zeigt an vier Beispielen aus dem Forum, dass derzeitige Problematiken keiner grundsätzlichen «Neuausrichtung der Medienpädagogik» bedürfen (S. 83). Zuerst wird der Forums-Vortrag «Freiheit und Kindheit im Netz» von Friederike Siller («YouCitizen. Kindheit und Freiheit im Netz», vgl. Siller im gleichen Band) skizziert und dahingehend kritisiert, dass der «Weitergabe tragender Prinzipien für Freiheit und Demokratie» zwar beigepflichtet werden kann, dass aber neben einem eher bewahrpädagogischen Ansatz (Bewahrung vor Schädlichem und Hinführung zu Wertvollem) auch eine handlungsorientierte Sichtweise zielführend wäre, innerhalb derer «Mediennutzung eine bedürfnisbezogene Handlung» und Medienhandeln «eine entwicklungsbezogene Aktivität» darstellt (S. 85). Auch bei der Bearbeitung der zweiten Thematik, «Big Data als Herausforderung für die Medienpädagogik», sucht Tulodziecki nach Anknüpfungspunkten in den Grundlagen der Medienpädagogik. Für besonders riskant hält er «eine grösstenteils funktionale Strukturierung von Medienkompetenz» und eine Reduktion von Medienkompetenz auf einen «Teil methodischer Kompetenz» (S. 88), bei der die inhaltlich-thematische Dimension von Medienbildung auf der Strecke bleibt. Die dritte Thematik, «Didaktische 


\section{Rezensionen}

Vorgehensweisen bei der Auseinandersetzung mit Datenstromanalysen» betrifft die medienpädagogische Arbeit mit Jugendlichen. Kritik wird dem Voranstellen eines «Aufreissers» zuteil, der sich als «blosses Mittel der Aufmerksamkeitsmanipulation» erweist, wohingegen das Voranstellen echter Aufgaben, welche «das Suchen nach Problemlösungen, das Treffen von Entscheidungen, eigene Gestaltungen oder Wertediskussionen herausfordern» für sinnvoller gehalten wird. Anhand des vierten Beispiels (dem Beitrag von Welling im gleichen Band über schulische Kommunikation) stellt Tulodziecki heraus, dass Medienpädagogik bei der Bearbeitung aktueller Problemlagen nicht «in erster Linie angebotsorientiert, sondern vor allem systemisch» und innovationsbezogen denken und handeln sollte (S. 96). Insgesamt wird durch den Beitrag deutlich, dass die Medienpädagogik mit ihren Grundlagen und Ansätzen bereits auf eigenen Füssen steht und in der Lage ist, auch für aktuelle Problemlagen Lösungen zu finden.

Thomas Knaus befasst sich in seinem Beitrag «digital - medial - egal? Ein fiktives Streitgespräch um digitale Bildung und omnipräsente Adjektive in der aktuellen Bildungsdebatte» mit dem «omnipräsenten» Adjektiv «digital», den er kritisch betrachtet. Dabei weist er zunächst darauf hin, dass in der medienpädagogischen Diskussion Chiffren wie «digitale Welten», «digitale Kultur» oder «digitale Bildung» eingeführt wurden. Diese werden seiner Auffassung nach zu wenig hinterfragt, unscharf definiert und eher intuitiv als reflektiert verwendet (S. 100). In Zukunft werden, so Knaus, ausgearbeitete Begriffe wie Medienkompetenz, Medienbildung etc. verdrängt (S. 102). Die Gefahren des Begriffswandels werden in einem fiktiven «Streitgespräch» zwischen einem Pädagogen, einer Bildungspolitikerin und einer Informatikerin dargestellt. Als Diskussionsgegenstand wählt Knaus die Schule (S. 104). Die Lesbarkeit des Gesprächs wird durch eine Auslagerung von Anmerkungen erreicht. In dem Gespräch werden Aspekte des Begriffs «Medienkompetenz», der in den letzten Jahrzehnten in der Pädagogik umfassend definiert wurde (S. 108), hervorgehoben und gleichzeitig die Befürchtung vor einer «inhaltlichen Verkürzung auf technische Fertigkeiten» durch den Begriffswandel geäussert ( $\mathrm{S}$. 107). Es folgen Überlegungen dazu, wie z.B. die Digitalisierung im Bildungssystem berücksichtigt werden kann (S. 112). Auf die Frage der Bildungspolitikerin, was «praktisch» getan werden solle, entwickelt sich ein Dialog zwischen Bildungspolitikerin und Informatikerin, bei welcher der Pädagoge zunächst aussen vor bleibt (S. 113ff.) Erst später kommt er zu Wort, um den Begriff Medienbildung zu erklären. Diese greifen seine Erklärungen auf und 


\section{Rezensionen}

reflektieren, wie die Überlegungen praktisch im Schulalltag umgesetzt werden können und Medienbildung praktisch implementiert werden kann (S. 117). Das Format des Kapitels als Gespräch ist sehr innovativ und kreativ. Knaus weist auf wichtige Aspekte hin, die mit dem Begriffswandel inhaltlich verbunden sind. Die Frage, ob die Bezeichnung «Streitgespräch» passt, darf offen bleiben. Die beteiligten Kommunikationspartner beziehen sich aufeinander, nehmen passend Argumente auf und können ihre Positionen ausführlich darstellen. Für einen «Streit» werden die Ausführungen des Pädagogen etwas zu wohlwollend aufgenommen. Das ändert allerdings nichts an dem wichtigen Impuls, den das Kapitel setzt. Neben den gravierenden Folgen eines einfachen Begriffswandels werden nebenbei wichtige Erkenntnisse der Medienpädagogik eingebracht, die durch diesen Begriffswandel in Vergessenheit zu geraten drohen.

Aßmann, Brüggen, Dander et al. befassen sich in ihrem sehr klar strukturierten Positionspapier mit der Bedeutung der Herausforderungen von Big Data für die Medienpädagogik. Sechs gesellschaftliche Problemfelder der Datafizierung werden umrissen. 1) Privatheit und Öffentlichkeit: Die zunehmende Verwischung von früher eindeutigen Grenzen der Privatsphäre durch digitale Überwachung, «Echokammern» und «Filter Bubbles» untergräbt eine ausgeprägte und demokratisch notwendige Meinungsvielfalt. 2) Informationelle Selbstbestimmung. Die Auswertung persönlicher Daten ermöglicht eine Vielzahl an Weiterverwendungsmöglichkeiten, über welche das Individuum nicht entscheiden kann. 3) Datenerhebungs- und Datenauswertungspraktiken. Die Strategien der Datenanalyse erlauben z.B. Rückschlüsse auf Gesundheit oder Konsumabsichten und können für Vorhersagen herangezogen werden (Predictive Analytics). 4) Datenkapitalismus. Digitale Informationen sind zur Grundlage globaler Konsum- und Finanzmärkte geworden. Daher steigt der Anreiz dafür, immer mehr Daten zu erheben und auszuwerten. 5) Menschliche Berechenbarkeit. Durch «Big Data Analytics» kann menschliches Verhalten berechnet werden. Durch die Zuschreibung von Handlungsabsichten oder Gruppenzugehörigkeiten aufgrund von Datenanalysen (Vorhersage von Straftaten mit Preditive Policing oder im Bildungskontext von Lernwegen mit Learning Analytics) werden Handlungs- und Willensfreiheit des Einzelnen eingeschränkt und es besteht die Gefahr der Entstehung neuer Diskriminierungen. 6) Mensch-Maschine-Verhältnis. Durch das Miteinander von miteinander kommunizierenden Menschen und teilweise ebenfalls autonom handelnden 


\section{Rezensionen}

Maschinen entstehen neue Fragen zum Verhältnis von Mensch und Maschine.

Zu den Aufgaben für die Medienpädagogik zählen: 1) Abstraktheit und Komplexität in Anschaulichkeit übersetzen. Es bedarf medienpädagogischer Forschung und praxistauglicher Methoden eines kritisch-reflexiven Umgangs mit Daten. Hierfür ist die Weiterentwicklung medienpädagogischer Konzepte zentral, z.B. Medienkritik, aktive Medienarbeit und journalistische Strategien. 2) Digitale Infrastrukturen durchschauen und demokratisch mitgestalten, gesellschaftliche Handlungsfähigkeit fördern, indem Menschen unterstützt werden, ihre Schutzbedarfe und Wünsche zu artikulieren und in Prozesse demokratischer Willensbildung einzubringen. So kann sichergestellt werden, dass Technologien entwickelt werden, die das Recht auf informationelle Selbstbestimmung berücksichtigen, z.B. durch die Umsetzung von Prinzipien wie «Privacy by Design» (Datenschutz per Technik). 3) Entwicklung diversitätsbewusster medienpädagogischer Didaktik und Methodik, um die Nicht-Berücksichtigung benachteiligter Gruppen in Bildungsangeboten und damit digitale Spaltung zu verhindern (Digital Divide). 4) Digitale Selbstbestimmung fördern, d. h. Verständigung über Schutz- und Einflussmassnahmen. 5) Gesellschaftlich wünschenswerte Nutzungsformen ermöglichen, z.B. Analyse von Open Data (nicht personenbezogener Daten) in Projekten aktiver Medienarbeit für gemeinnützige Zwecke.

Friederike Siller beschreibt in ihrem Beitrag "YOUCitizen. Kindheit und Freiheit im Netz» die wachsende Bedeutung der Medienpädagogik für gesellschaftliche Fragen zu Demokratie und Freiheit, die auch Kindern zugänglich gemacht werden sollten. Um Kindern Freiheit zu ermöglichen, müssen innen einerseits Erprobungsräume für soziale Vernetzung zugestanden und andererseits gleichzeitig Unterstützung angeboten werden, z.B. mit der Eröffnung von Parallelwelten, in denen wichtige politische Informationen kindgerecht dargestellt werden.

Stefan Wellings Beitrag mit dem Titel «Die schulische Kommunikation des Organisierens im Spiegel von Medienwandel und Mediatisierung» ist für eine Leserschaft, die Konzepte und Strategien der Medienpädagogik und Medienbildung erwartet, zunächst unbefriedigend, denn weder das eine noch das andere ist sein Thema. Vielmehr blickt Welling aus einer an Schulentwicklungsprozessen interessierten Perspektive, wie sie die Educational Governance-Forschung als bildungsbezogene Organisationsforschung praktiziert, 


\section{Rezensionen}

darauf, wie sich die Kommunikation von Lehrkräften eines Kollegiums jenseits des Unterrichts durch Mediatisierung und Medienwandel in den letzten Jahren verändert hat. Dies stellt er in den Kontext der Debatte um Schulautonomie und Ökonomisierung des Bildungssystems durch die Implementierung von Methoden neuer Steuerung (siehe dazu Geiss \& De Vincenti, 2012). Kommunikation versteht er im Sinne Habermas' als kommunikatives Handeln, welches «die Organisation Schule im Prozess des Organisierens» konstituiere (S. 155). Nonverbale Kommunikation taucht in seinem Modell nicht auf, es geht ausschliesslich um sprachlich oder textuell via analoger und/oder digitaler Medien vermittelte Kommunikationsprozesse. Wie und wo diese stattfinden, beschreibt er ab Seite 161 im Rückgriff auf die Ergebnisse einer in 2011/12 an zwei Gesamtschulen durchgeführten Studie (Welling, Breiter, und Schulz 2015) und stellt fest: «Papier hat bislang kaum etwas von seiner Relevanz für die schulische Kommunikation des Organisierens verloren» (S. 163), digitale Schulinformationssysteme sind «kaum relevant» (S. 164), einzig die digitale Kommunikation via E-Mails konnte sich an den untersuchten Schulen etablieren (S. 165). Ausgehend von seinen Erkenntnissen über die Praktiken digitaler Kommunikation zwischen Lehrkräften macht Welling Aussagen über mediengestützte Lernprozesse. Das ist kritisch zu betrachten, denn bei Lernprozessen - seien sie nun mediengestützt oder nicht - handelt es sich um komplexe Phänomene, die nicht mit Abläufen kommunikativer Schulorganisation vergleichbar sind. Unscharf bleiben auch die Verhältnisse zwischen dem Konzept des kommunikativen Handelns und praxeologischen (Helsper) bzw. diskurstheoretischen Ansätzen (Böhme), was der Kürze des Beitrags geschuldet sein mag. Sein Ansatz ist insofern ertragreich, als er verdeutlicht, dass ein wie auch immer gearteter, schulinterner Einsatz digitaler Medien in einem von verschiedenen Akteuren, Technologien, Einstellungen, Diskursen und Praktiken geprägten Raum stattfindet (siehe dazu die Ausführungen von Böhme (2009) zum «Spatial Turn») und nicht in einer Tabula rasa.

Katrin Valentin bezieht sich auf das Praxisfeld der Kinder- und Jugendarbeit und die pädagogische Arbeit darin. Diese kann als ein Austarieren zahlreicher Spannungsverhältnisse beschrieben werden, z.B. zwischen Nähe und Distanz. Ändern sich durch den Prozess der Digitalisierung der Lebenswelten die Bezugspunkte, zwischen denen die Spannungen entstehen, bedarf es der Entwicklung neuer Handlungsschemata, welche zwischen Klientel und Fachkräften neu ausgehandelt werden müssen. Dies beantwortet insofern die eingangs gestellte Frage, ob die Kinder- und Jugendarbeit den Anschluss an die 


\section{Rezensionen}

(digitale) Lebenswelt inrer Zielgruppen verliert, als deutlich wird, dass sich durch den Mediatisierungsschub ein tiefgreifender Wandel vollzieht. Diesen als ein »sprach- und handlungsfähiges Gegenüber» zu begleiten und zu gestalten, ist zu einer grundlegenden Aufgabe der Kinder- und Jugendarbeit geworden, was in diesem Beitrag deutlich herausgearbeitet wird.

In ihrem Beitrag «There are no girls on the internet. Gender und Kommunikation in Online-Gaming-Szenen» beabsichtigen Maike Groen und Tanja Witting einen vielschichtigen Erklärungsansatz dafür zu bieten, warum und wie Frauen aus bestimmten Bereichen von Online-Communities ausgeschlossen werden. Hierfür thematisieren sie zunächst kurz Medien im Sozialisationsprozess und das Erlernen bzw. Aktualisieren normierter Geschlechterrollen im Kontext von Spielzeug und Spielen. Dabei erläutern sie, wie Sexismus im Rahmen von Sozialisation zur Entwicklung bzw. Verstärkung geschlechtsspezifischer Spielinteressen führt. Sie kritisieren zudem androzentrierte Fragestellungen bei der Mediennutzungsforschung, die weiblich codiertes Spielverhalten ausblenden und Ähnlichkeiten zwischen Jugendlichen verschleiern. Auf Grund des verbreiteten Andozentrismus in Kombination mit der Marginalisierung von Spielerinnen werden insbesondere junge Männer als Hauptzielgruppe von digitalen Spielen wahrgenommen, weshalb die Entwicklungsstudios Marketing und Design auf diese Gruppe ausrichten und so entsprechende Produkte noch unattraktiver für Mädchen und Frauen machen. Unter der Überschrift «Vergeschlechtlichtes Spieldesign: Sexismus als Marketingeffekt» thematisieren die Autorinnen die Chance, mittels digitaler Spiele alternative Geschlechterrollen zu erlernen und stellen zugleich fest, dass entsprechende Angebote gerade im Bereich der kommerziell erfolgreichsten Titel sehr begrenzt sind. Dies führen sie auf die Zielgruppenorientierung sowie die Dominanz von Männern im Produktionsprozess digitaler Spiele zurück. Das Ergebnis ist die Reproduktion dichotomer Geschlechterverhältnisse und stereotyper Darstellungen von Männern sowie Frauen entlang hegemonialer Herrschaftslinien und damit Spielinhalte, die von heterosexuellen, weissen Männern geprägt sind. Unter der Überschrift «Kommunikationskulturen in Communities: Sexismus als alltägliche soziale Praxis» benennen sie die Marginalisierung von Frauen und Minderheiten wie Homosexuellen in Werbung und Events der Gaming-Szene und fokussieren dann Belästigungen, Beleidigungen, Hate Speech und Toxic Behavior gegenüber Spielerinnen innerhalb der Gaming Community. Dies veranschaulichen die Autorinnen an einzelnen Beispielen u.a. aus dem Bereich E-Sport. Abschliessend konstatieren sie, 


\section{Rezensionen}

dass Freizeitaktivitäten das Ergebnis komplexer, ressourcenabhängiger relativer Positionierungen im sozialen Raum sind und verweisen auf die Relevanz von sowie den Mangel an pädagogischen Projekten, die sich explizit mit Gender-Aspekten in digitalen Spielen auseinandersetzen. Insgesamt geben die Autorinnen einen nachvollziehbaren ersten Einblick in das brisante und aktuelle Thema des Sexismus im Kontext digitaler Spiele und zugehöriger Communities, welches u.a. durch die unter dem Hashtag \#Gamergate geführte, hochemotionale und zum Teil erbitterte Debatte öffentliche Aufmerksamkeit erlangte (Stoltenhoff und Raudonat 2016).

Der Beitrag «Hörkultur im digitalen Zeitalter - eine medienpädagogische Perspektive» von Schill und Pöttinger greift einleitend den Begriff des Hörens und den Digitalisierungsbegriff auf. Für den Beitrag wurde eigens eine «Toncollage» erstellt, die über einen Link abgerufen werden kann. Es werden Veränderungen der Geräuschkulisse allgemein im Zuge gesellschaftlicher Veränderungen angesprochen (z.B. das Verschwinden des Rasselns von Supermarktkassen) sowie Veränderungen von auditiven Medien (z.B. das Abnehmen der Bedeutung von Schallplatten oder Walkmans und die zunehmende Verbreitung von MP3-Playern oder Smartphones). Nach der knappen Auseinandersetzung mit diesen und weiteren theoretischen Aspekten folgt eine Vorstellung der ARD-Radionacht als gelungenem Event, das Kinder zu weiteren aktiven Medienprojekten anregen kann. Anhand des Beispiels der Radionacht wird weiter reflektiert, auf welchen Ebenen Teilnehmende durch ein solches medienpädagogisches Projekt angeregt und gefördert werden. Auf den letzten Seiten (S. 205ff.) folgt eine kurze Beschreibung von medienpädagogischen Angeboten im Internet, die sich vorwiegend an pädagogische Fachkräfte im Kindertagesstätten- und Schulbereich sowie direkt an Kinder richten.

Im nächsten Beitrag (Inklusive Medienbildung und «Werkstatt einfache Sprache») beschreiben Palme und Staufer die Inklusionsperspektive als zentralen Blickwinkel für die Medienpädagogik anhand von Beispielen für inklusive Medienprojekte. Da Prozesse gesellschaftlicher Partizipation und Informationsgewinnung heute immer mehr online stattfinden, können medienpädagogische Angebote so gestaltet werden, dass sie der Aufhebung struktureller Benachteiligung und der chancengleichen Teilhabe aller Interessierten dienen. Ziel inklusiver Medienarbeit ist ein Empowerment durch Medien. Am Beispiel einer QR-Code-Rallye werden die Prinzipien aktiver Medienarbeit, Lebens- 


\section{Rezensionen}

welt-, Handlungs- und Ressourcenorientierung dargestellt. Die Rolle «einfacher Sprache» im Prozess der Verständigung, also leicht lesbarer und verständlicher Sprache, wird am Modell der «Werkstatt einfache Sprache» und anhand aktueller Beispiele aus der Reihe «einfach Politik» der Bundeszentrale für politische Bildung $(\mathrm{BpB})$ erläutert.

Uwe Hasebrink geht in seinem Beitrag (Medienkompetenz in Europa. Vom Nutzen international vergleichender Forschung) der Frage nach, inwiefern Medienkompetenz von Kindern und Jugendlichen kulturell beeinflusst ist und inwiefern international vergleichende Forschung zu einem besseren Verständnis der Förderung von Medienkompetenz beitragen kann. Die «besonderen konzeptionellen und forschungsökonomischen Herausforderungen» international vergleichender Forschung tragen zu einer eher schwachen theoretischen und empirischen Grundlage hinsichtlich kulturübergreifend verallgemeinerbarer Befunde und länderspezifischer Besonderheiten bei (S. 229). Die Schwierigkeiten international vergleichender Forschung liegen in der Unterschiedlichkeit der Vergleichsländer sowie der Vernachlässigung von Kontextfaktoren bei der Beschreibung von Gemeinsamkeiten und Unterschieden zwischen den Ländern. Methodische Probleme sind der Beschaffenheit der Untersuchungsdesigns, der Stichproben sowie der Übersetzung der Forschungsinstrumente geschuldet. Fortschritte hat dabei das europäische Forschungsnetzwerk «Safer Internet Programme» der Europäischen Kommission mit einer in 25 europäischen Ländern durchgeführten Studie zu Onlinenutzung und Onlinerisiken erzielt. An der Untersuchung nahmen 25.142 Kinder und Jugendliche im Alter zwischen 9 und 16 sowie ein Elternteil teil. Erhoben wurden Häufigkeit und Dauer von Internetanwendungen, Umgang mit sozialen Netzwerken, persönlichen Daten und negativen Erlebnissen sowie elterliches Erziehungsverhalten und die Nutzung von Jugendschutzsoftware. Hasebrink unterscheidet wie Kohn (1989) vier Typen vergleichender Forschung, 1) «Einzelländer als Untersuchungsobjekte», 2) ländervergleichende Perspektive, 3) «Länder als Kontexte zur Überprüfung von Universalität von Hypothesen» und 4) «Länder als Bestandteile grösserer transnationaler/globaler Systeme». Beim zweiten Forschungstyp werden bestimmte Variablen über verschiedene Länder hinweg untersucht und verglichen. Bei der Befragung von EU Kids Online wiesen deutsche Kinder und Jugendliche durchschnittlich die Hälfte von acht onlinebezogenen Fähigkeiten auf, während 17 Länder höhere Werte und nur sieben niedrigere Werte aufwiesen. Mit dem 


\section{Rezensionen}

dadurch entstehenden Benchmarking-Effekt sind höhere Chancen verbunden, in der Politik Gehör für Massnahmen zur Förderung von Medienkompetenz zu finden. Durch die Untersuchung von Kontextfaktoren und der universellen Gültigkeit bestimmter theoretischer Annahmen (dritter Forschungstyp) wird ein besseres Verständnis der Rolle von Medienkompetenz, was Empfehlungen für Massnahmen zur Förderung von Medienkompetenz ermöglicht. Der Fokus des Beitrags liegt auf einer «ländervergleichenden Perspektive im engeren Sinne». Der vierte Forschungstyp der Untersuchung transnationaler Phänomene erfährt keine weitere Vertiefung, welche jedoch z.B. für Fragestellungen hinsichtlich weltweit genutzter Dienste wie WhatsApp oder Facebook interessant wäre.

Ida Pöttinger und Sebastian Ring (Medienpädagogik in Europa. Vier Einrichtungen - eine Zielrichtung?) befassen sich mit Ergebnissen der European Session des 32. Forums Kommunikationskultur in Köln, bei der es um Projekte, Initiativen und Vernetzungsansätze auf europäischer Ebene ging. Es wird betont, dass aktuellen Herausforderungen gerade auf internationaler Ebene begegnet werden muss. Dabei sei strukturelle Vernetzung notwendig, wenn vermieden werden soll, dass auf politischer Ebene Entscheidungen getroffen werden, welche medienpädagogische Argumente ausser Acht lassen. Vorgestellt werden vier Akteure mit unterschiedlichen Traditionen und der Gemeinsamkeit medienpädagogischer Kooperation auf europäischer/ internationaler Ebene: 1) das CLEMI in Frankreich (Centre de Liaison de l'Enseignemnet et des Médias d'Information), einer Abteilung des französischen Unterrichtsministeriums, welches für die verbindliche Gestaltung der medienpädagogischen Lehrpläne verantwortlich ist und an der Entstehung der Medienpädagogischen Charta beteiligt war (Charter for Media Literacy, www.euromedialiteracy.eu), 2) die Evens Foundation (ef), einer privaten Stiftung, welche europaweit um die Themen Peace Education, European Citizenship und Medienpädagogik bemüht ist, einen Preis für europäische Medienpädagogik verleiht und zwei hilfreiche (auch downloadbare) Broschüren aufgelegt hat («Media literacy in Europe: 12 good practices that will inspire you» sowie «Inspiring ways to involve parents", 3) die EAVI (European Association for European Viewers), welche auch in internationalen Kontexten operiert und kreative Videoclips zur Vermittlung von Medienkompetenz in mehreren Sprachen erstellt (z.B. «A Journey to Media Literacy Episode 2: Awareness», https://www.youtube.com/watch?v=9nUdvBL7 iU) und 4) das Hans-Bredow-Institut, an dem seit 2001 internationale Studien durchgeführt werden, u. a. die auch in 


\section{Rezensionen}

diesem Band von Uwe Hasebrink vorgestellte Studie «EU Kids Online - Kinder und Internetnutzung» sowie das Projekt «Identifikation von Good Practice im Jugendmedienschutz im internationalen Vergleich» von Schulz et al. (2015). Neben den genannten Leuchtturm-Beispielen beschreiben die Autoren auch die mit europäischer und internationaler Kooperation verbundenen Schwierigkeiten: nicht nur müssen Kontakte gepflegt werden, was im Alltag oft schwierig ist, es kommen auch Sprachbarrieren hinzu sowie unterschiedliche Perspektive auf die Frage, was europäische Medienpädagogik ausmacht.

Insgesamt betrachtet gibt dieser Band einen guten Einblick in verschiedene Perspektiven auf aktuelle medienpädagogische Fragestellungen und beleuchtet diese anhand von konkreten Projekten aus der Praxis. Die einzelnen Beiträge unterscheiden sich stark in ihrer Schwerpunktsetzung, z.B. richten sich einige eher an Praktikerinnen und Praktiker, welche an der theoretischen Fundierung ihrer Arbeit interessiert sind. Andere Beiträge sind eher wissenschaftlichen Charakters. Trotz der Heterogenität der Beiträge ist den Herausgeber*innen eine kompakte Zusammenstellung gelungen, welche die aktuellen Fragestellungen zur Sprache bringt, an welchen sich die Medienpädagogik orientieren und gleichzeitig Orientierung bieten soll.

Annette Ullrich, Sven Trabandt, Ann-Kathrin Stoltenhoff, Kerstin Raudonat

\section{Literatur}

Böhme, Jeanette. 2009. Raumwissenschaftliche Schul- und Bildungsforschung. In Jeanette Böhme. Hrsg. Schularchitektur im interdisziplinären Diskurs. Territorialisierungskrise und Gestaltungsperspektiven des schulischen Bildungsraums, S. 13-22. Wiesbaden: VS Verlag für Sozialwissenschaften.

Geiss, Michael, und Andrea De Vincenti. Hrsg. 2012. Verwaltete Schule. Geschichte und Gegenwart. Wiesbaden: VS Verlag für Sozialwissenschaften.

Stoltenhoff, Ann-Kathrin, und Kerstin Raudonat. 2016. Medienpädagogik im Spannungsfeld der (Re)Produktion heteronormativer Machtstrukturen und emanzipatorischer Bildungsideale - Eine poststrukturalistische Perspektive. [Macht, Souveränität, Herrschaft]. Medienimpulse, 4, http://www.medienimpulse.at/articles/view/1010. 